\title{
Association between dietary saturated fat with cardiovascular disease risk markers and body composition in healthy adults: findings from the cross-sectional BODYCON study
}

Ezgi Ozen, Rada Mihaylova, Michelle Weech, Sam Kinsella, Julie A. Lovegrove and Kim G. Jackson* (1)

\begin{abstract}
Background: Diets high in saturated fatty acids (SFAs) and greater abdominal obesity are both associated with raised low-density lipoprotein cholesterol (LDL-C) concentrations, an independent cardiovascular disease (CVD) risk marker. Although reducing SFA intake is a public health strategy for CVD prevention, the role of body fat distribution on the relationship between SFA and LDL-C is unclear. Therefore, our objective was to investigate whether the association between dietary SFAs and LDL-C concentrations is related to body composition.

Methods: In the BODYCON (impact of physiological and lifestyle factors on body composition) study, 409 adults [mean age $42 \pm 16$ years and median BMl of $23.5(21.5-25.9) \mathrm{kg} / \mathrm{m}^{2}$ ] underwent a measure of body composition by dual energy $\mathrm{x}$-ray absorptiometry, assessment of habitual dietary intake using a 4-day weighed food diary and physical activity level using a tri-axial accelerometer. Blood pressure was measured, and a fasting blood sample was collected to determine cardiometabolic disease risk markers. Correlations between body composition, circulating risk markers and dietary macronutrients were assessed prior to multivariate regression analysis. The effect of increasing intakes of dietary SFA on outcome measures was assessed using ANCOVA after adjusting for covariates.

Results: Abdominal visceral adipose tissue (VAT) mass was moderately positively correlated with total cholesterol (TC), LDL-C, systolic blood pressure (SBP), diastolic blood pressure and HOMA-IR $\left(r_{s}=0.25-0.44, p<0.01\right)$. In multiple regression analysis, $18.3 \%$ of the variability in LDL-C was explained by SFA intake [\% total energy (TE)], abdominal VAT mass, carbohydrate\%TE and fat\%TE intakes. When data were stratified according to increasing SFA\%TE intakes, fasting TC, LDL-C and non-high-density lipoprotein-cholesterol were higher in Q4 compared with Q2 ( $p \leq 0.03)$. SBP was higher in Q4 versus Q3 ( $p=0.01$ ). Android lean mass was also higher in Q3 versus Q1 ( $p=0.02)$. Other anthropometric and CVD risk markers were not different across quartile groups.
\end{abstract}

${ }^{*}$ Correspondence: k.g.jackson@reading.ac.uk

Hugh Sinclair Unit of Human Nutrition, Department of Food

and Nutritional Sciences and Institute for Cardiovascular and Metabolic

Research and Institute for Food, Nutrition and Health, University

of Reading, Harry Nursten Building, Whiteknights, Pepper Lane,

Reading RG6 6DZ, UK

(c) The Author(s) 2022. Open Access This article is licensed under a Creative Commons Attribution 4.0 International License, which permits use, sharing, adaptation, distribution and reproduction in any medium or format, as long as you give appropriate credit to the original author(s) and the source, provide a link to the Creative Commons licence, and indicate if changes were made. The images or other third party material in this article are included in the article's Creative Commons licence, unless indicated otherwise in a credit line to the material. If material is not included in the article's Creative Commons licence and your intended use is not permitted by statutory regulation or exceeds the permitted use, you will need to obtain permission directly from the copyright holder. To view a copy of this licence, visit http://creativecommons.org/licenses/by/4.0/. The Creative Commons Public Domain Dedication waiver (http://creativeco mmons.org/publicdomain/zero/1.0/) applies to the data made available in this article, unless otherwise stated in a credit line to the data. 
Conclusions: Although dietary SFA was found to explain 9\% of the variability in LDL-C, stratification of data according to quartiles of SFA intake did not reveal a dose-dependent relationship with LDL-C concentration. Furthermore, this association appeared to be independent of abdominal obesity in this cohort.

Clinical Trail registration: Trial registration: clinicaltrials.gov as NCT02658539. Registered 20 January 2016, https://clini caltrials.gov/ct2/show/NCT02658539.

Keywords: Body composition, Abdominal obesity, Dietary fat quality, SFA intake, DXA

\section{Introduction}

Diet is one of the most important modifiable risk factors for cardiovascular diseases (CVDs), with studies reporting a link between high intakes of dietary saturated fatty acids (SFAs) and elevated low-density lipoprotein-cholesterol (LDL-C), a well-documented independent risk factor for this disease [1, 2]. Although many studies have investigated the effect of reducing dietary SFA intake on the fasting lipid profile, replacement with unsaturated fatty acids was found to be more beneficial compared to carbohydrates or protein $[3,4]$. Thus, current UK recommendations for CVD prevention are to decrease dietary SFA intake to less than $10 \%$ of total energy (TE) via replacement with polyunsaturated (PUFAs) and monounsaturated fatty acids (MUFAs) [5]. However, there is also consistent evidence suggesting no beneficial effect of reducing dietary SFA intake on CVD mortality [6-8]. These discrepancies between studies indicate that there may be other factors affecting this relationship.

Obesity is a rapidly growing global public health problem affecting over one third of the world's population [9, 10]. An excessive accumulation of body fat is positively associated with the risk of cardiometabolic diseases such as CVD and type 2 diabetes [11]. Body mass index (BMI) has been used routinely at a population level to assess adiposity and identify people with increased metabolic disease risk. However, body fat distribution is now considered to be a better indicator of chronic disease risk than BMI, with fat accumulation in the abdominal area [especially visceral adipose tissue (VAT)] associated with greater CVD risk compared with gynoid adiposity [12-15]. Moderately elevated LDL-C concentrations and insulin resistance have been observed in people with increased abdominal fat accumulation [16-18]. As a result, there is a considerable interest in the physiological and lifestyle characteristics that influence body fat distribution $[19,20]$.

Storage of body fat is influenced by non-modifiable factors such as age and sex [21], but also by modifiable lifestyle factors such as diet [22]. Studies have investigated the effect of dietary fat quality on body composition, with differential associations shown between dietary SFA (positive) and PUFA/MUFA (negative) with abdominal obesity [23-25]. Although the impact of dietary SFAs on LDL-C concentrations has been shown in many studies, the effect of body composition on this relationship is poorly understood. A small number of studies have reported BMI to be inversely associated with the LDL-C response to reduced SFA intake [26]. As dietary SFAs are reported to influence both LDL-C concentrations and body composition, the effect of dietary SFAs on LDL-C, therefore, might be related to its effect on body fat content and distribution.

Thus, the purpose of this study was to investigate whether the impact of dietary SFA on LDL-C was associated with body composition. We hypothesized that higher SFA intakes are related to increased LDL-C concentrations due to greater fat accumulation in the abdominal area.

\section{Methods \\ Subjects}

Healthy men and women $(n=409)$ aged $18-70$ years were recruited from Reading and the surrounding area (UK), from 2014 through 2019 using posters, pamphlets and by contacting previous volunteers registered on the Hugh Sinclair Unit of Human Nutrition volunteer database at the University of Reading. A Medical and Lifestyle questionnaire was used to assess the suitability of interested volunteers before potentially eligible individuals were invited to attend a screening session in which they were provided with detailed information about the study before signing a consent form. All subjects were assessed after fasting overnight for $12 \mathrm{~h}$. During the screening visit, blood pressure and anthropometric measurements were taken and a fasting blood sample was collected for the measurement of fasting blood lipids [total cholesterol (TC), triacylglycerol (TAG) and high density lipoprotein cholesterol (HDL-C)], glucose, kidney and liver function markers (alkaline phosphatase, alanine aminotransferase, $\gamma$-glutamyl transferase, serum creatinine, total bilirubin and uric acid) by using the ILAB 600 clinical chemistry analyser (Werfen Ltd, Warrington,UK). To determine the haemoglobin level, a further blood sample was sent to the Royal Berkshire Hospital Pathology Department (Reading, UK). All participants whose screening measurements matched the following inclusion criteria were invited to participate in the study: BMI $18.5-39.9 \mathrm{~kg} / \mathrm{m}^{2}$, 
TC $<7.8 \mathrm{mmol} / \mathrm{l}$, TAG $<2.3 \mathrm{mmol} / \mathrm{l}$, fasting blood glucose $<7.8 \mathrm{mmol} / \mathrm{l}$, haemoglobin $>115 \mathrm{~g} / \mathrm{l}$ for women and $130 \mathrm{~g} / \mathrm{l}$ for men. Exclusion criteria included the following: having suffered a myocardial infarction/stroke in the past 12 months, history of diabetes or other endocrine disorders, bowel disease, cholestatic liver disease, pancreatitis, cancer, being on medication for hyperlipidemia, hypertension, inflammation or hypercoagulation, being on a weight reducing diet and excessive alcohol consumption $(<14$ units/wk). Furthermore, due to the use of the dual energy $x$ ray absorptiometry (DXA) to assess body composition, further exclusion criteria included arthritis or fracture deformity of spine or femur, history of bone related surgeries, radio-opaque implants or implanted medical devices. Females were also excluded if they were breast feeding, may be pregnant or planning a pregnancy in the next 12 months.

\section{Study design}

Impact of physiological and lifestyle factors on body composition (BODYCON) was a single-centered observational cross-sectional study conducted in the Hugh Sinclair Unit of Human Nutrition at the University of Reading. The NHS and University of Reading Research Ethics Committees (reference numbers 14/SC/1095 and $13 / 55$, respectively) both gave a favorable ethical opinion for conduct. This study was carried out in accordance with the Declaration of Helsinki and was registered at www.clinicaltrials.gov (NCT02658539).

Participants attended a single study visit. For the day prior to this visit, participants were requested to abstain from strenuous exercise and consuming alcohol. A lowfat evening study meal and low-nitrate water (Buxton mineral water, Nestlé waters, UK) were provided by the researchers and participants were asked not to consume anything apart from this water after their evening meal. Before starting the study visit, a spot urine sample was collected and urine osmolarity was measured using an Osmocheck device (Vitech Scientific Ltd., UK) to ensure participants were sufficiently hydrated for the body composition measurements and asked to complete a pre-DXA scan questionnaire. Weight, waist and hip circumferences were measured, followed by clinic blood pressure. Total body composition was assessed by DXA scan before a fasting blood sample was taken to measure cardiometabolic disease risk markers. Additionally, in the few days before their visit participants were asked to complete a 4-day weighed food diary for 3 consecutive weekdays and 1 weekend day while wearing a triaxial Actigraph activity monitor (ActiGraph, Florida, US) during the same time to assess dietary intake and physical activity levels, respectively. Premenopausal women not taking oral contraceptives attended their main study visit during the same phase of their menstrual cycle (days $1-7)$.

\section{Anthropometric and blood pressure measurements}

Anthropometric and body composition measurements were performed with participants wearing light clothing and no shoes or metal objects. Height was measured to the nearest $1 \mathrm{~cm}$ using a stadiometer, facing forwards, and standing as straight as possible with their arms hanging loosely by their side and their head in the Frankfort plane. Body weight and BMI were determined by using a bioelectrical impedance analyser (Tanita BC-418, TANITA UK Ltd, Middlesex, UK) and $1 \mathrm{~kg}$ was automatically deducted to account for the weight of the subject's light clothing. Waist circumference (WC) was measured at the midpoint between the lowest ribs and the top of the iliac crest while hip circumference was measured at the largest circumference around the buttocks. Both measurements were taken by a trained researcher while participants were standing straight after a gentle expiration. A non-stretch tape measure (Seca, UK) was used for both measures. The waist to hip ratio (WHR) and waist to height ratio (WHtR) were calculated as estimates of body fat distribution.

Blood pressure was measured three times using an Omron blood pressure monitor (Omron M3 digital automatic upper arm blood pressure monitor, Omron Healthcare Co UK Ltd.) and the average systolic blood pressure (SBP) and diastolic blood pressure (DBP) were calculated. Pulse pressure was determined by subtracting DBP from SBP.

Visceral adiposity, fat mass and lean mas index calculations Anthropometric indices were calculated to determine their relationship with dietary SFA and cardiometabolic disease risk markers. These included the visceral adiposity index $(\mathrm{VAI}=$ waist circumference $/(39.68+(1.88 \times \mathrm{BM}$ I) $\times($ TAG $(\mathrm{mmol} / \mathrm{L}) / 1.03) \times(1.31 / \mathrm{HDL}-\mathrm{C}(\mathrm{mmol} / \mathrm{L}))$ for men and $\mathrm{VAI}=$ waist circumference $/(36.58+(1.89 \times \mathrm{BM}$ $\mathrm{I})) \times(\mathrm{TAG}(\mathrm{mmol} / \mathrm{L}) / 0.81) \times(1.52 / \mathrm{HDL}-\mathrm{C}(\mathrm{mmol} / \mathrm{L}))$ for women as an indicator of visceral adipose tissue function [27]), fat mass index $\left(\mathrm{FMI}=\right.$ fat $\operatorname{mass}(\mathrm{kg}) /$ height in $\left.\mathrm{m}^{2}\right)$ and lean mass index $\left(\mathrm{LMI}=\right.$ lean mass $(\mathrm{kg}) /$ height in $\left.\mathrm{m}^{2}\right)$ [28].

\section{Assessment of dietary intake}

Habitual dietary intake was evaluated by using a 4-day weighed diet diary. To increase accuracy, an electronic kitchen scale and a selection of food portion sizes from the Food Atlas to record meals consumed outside of home [29] were provided to the participants. Instructions on how to complete the diary were given both verbally and in written form by the researchers. For each 
subject, nutrient and energy intakes were calculated using Dietplan 7 (Forestfield Software) and the total dietary intakes were divided by the number of days recorded to give mean daily intakes. Data entered on Dietplan was checked by a single researcher at the end of the study. For dietary data inclusion, participants were required to complete at least 3 days of the diet diary and report feasible dietary intakes between 500 and $3500 \mathrm{kcal}$ per day for women and 800 and $4000 \mathrm{kcal}$ per day for men. Individuals with dietary intakes outside of these ranges have been previously reported to be under and over reporters [30].

\section{Physical activity}

A tri-axial accelerometer was used to measure physical activity levels (Actigraph wGT3X+, Actigraph, LLC). Participants were asked to wear the accelerometer for 4 consecutive days including 3 weekdays and 1 weekend day and keep an activity diary for data cleaning purposes. It was worn around the abdomen above their right hip bone, and they were asked to remove the device only for showering or during swimming. Device initialization, data processing and analysis were conducted using Actilife Data Analysis Software (Version 6.11.5) as previously described [31]. Raw data was collected at a $30 \mathrm{~Hz}$ sample rate. For inclusion in the physical activity analysis, participants were required to have produced counts on their activity monitor for $\geq 3$-days ( $>600 \mathrm{~min} /$ day of wear time) [32]. Non-wear-time was defined as $\geq 60 \mathrm{~min}$ of zero activity counts [33]. Data were summarized in 60-s epochs and cut-points were used to classify wear time as: sedentary behaviour $(<100$ counts/min), light/lifestyle physical activity (760-1951 counts/min), moderate physical activity (1952-5724 counts/min) and vigorous physical activity ( $\geq 5725$ counts/min) [34]. For the purposes of the data analysis, the time spent in moderate and vigorous physical activity was combined. Mean energy expenditure from physical activity $\left(\mathrm{EE}_{\mathrm{PA}}\right)$ was calculated as $\mathrm{kcal} /$ day.

\section{Details of the DXA procedure}

Prior to the DXA scan assessment, participants changed into clothing without zips and metal buttons or a disposable hospital garment and all metal artefacts were removed. Whole body composition was measured by Lunar iDXA (GE Healthcare, UK) and two operators performed the scanning and followed the manufacturer's guidelines for volunteer positioning and for scan acquisition. Participants laid supine on the Lunar iDXA scanning table with knees and ankles positioned together using the Lunar Velcro supports. Arms were positioned to the side of the body, with palms facing towards the body and participants were required to lie still during the total body composition scan. All scans were analysed using enCORE Software, version 15 (GE Healthcare, UK) with the advance software package CoreScan, which also estimates the mass and volume of visceral fat within the abdomen. The machine's performance was checked daily by running a quality assurance test according to the manufacturer's instructions before each scanning session.

\section{Biochemical analysis}

Blood samples collected into the serum separator and $\mathrm{K}_{3}$ EDTA blood tubes were centrifuged at $1700 \times g$ (3000 rpm) for $15 \mathrm{~min}$ at room temperature and $4{ }^{\circ} \mathrm{C}$, respectively before aliquoting into Eppendorf tubes and stored at $-20{ }^{\circ} \mathrm{C}$. Fasting serum lipids (non-esterified fatty acids (NEFA) (Alpha Laboratories Ltd., Hampshire, UK), TC, HDL-C and TAG), glucose, C-reactive protein (CRP), and y-glutamyl transferase (GGT) were quantified in the main study visit sample by using the ILAB 600 clinical chemistry analyser with reagents from Werfen (Werfen (UK) Ltd., Warrington, UK). Plasma uric acid was measured using RX Daytona Plus clinical chemistry analyser (Randox Laboratories Ltd., County Antrim, UK) using a kit supplied by Randox. The Friedewald formula was used to estimate fasting LDL-C concentrations [35]. Non-HDL-C was calculated by subtracting HDL-C from TC. ELISA kits were used to analyse serum insulin (Dako Ltd., High Wycombe, UK) and plasma adiponectin (Quantikine kit, R\&D Systems, Europe Ltd.) concentrations. Homeostatic model assessment for insulin resistance (HOMA-IR) was calculated by using the following equation: [fasting insulin $(\mathrm{pmol} / \mathrm{l}) \times$ fasting glucose $(\mathrm{mmol} / \mathrm{l})] / 135$ [36]. Serum 25 hydroxyvitamin $\mathrm{D}_{2}$ and 25 hydroxyvitamin $\mathrm{D}_{3}$ was measured by the LGC group (LGC Ltd., Middlesex, UK) and summed to obtain total 25 hydroxy vitamin D $(25(\mathrm{OH}) \mathrm{D})$.

\section{Statistical analysis}

Statistical analyses were performed using IBM SPSS Statistics version 25 (SPSS Inc., IL, US). Data was presented as mean \pm standard deviation (SD) for normally distributed variables and as median (interquartile range) for non-normally distributed variables in Tables 1 and 2 . Normality was assessed using the Kolmogorov-Smirnov test and Q-Q plots. The logarithms or square root transformations were used for several outcome measures including BMI, body fat mass, abdominal VAT mass, dietary protein and trans-fat, TAG, LDL-C: HDL-C ratio, TC: HDL-C ratio, NEFA, CRP, GGT, adiponectin, insulin and HOMA-IR, steps/day, $\mathrm{EE}_{\mathrm{PA}}$, and percentage time spent performing moderate to vigorous physical activity. Parametric independent sample $t$ tests were used for normally distributed and transformed data to determine the differences between the male and female groups. Spearman's correlations were used to analyse relationships 
Table 1 Characteristics of BODYCON study participants

\begin{tabular}{|c|c|c|c|c|}
\hline & All $(n=409)$ & Men $(n=190)$ & Women $(n=219)$ & $p$ value $^{\mathrm{a}}$ \\
\hline \multicolumn{5}{|l|}{ Outcome measures ${ }^{b}$} \\
\hline Age, years & $42 \pm 16$ & $42 \pm 15$ & $42 \pm 16$ & 0.93 \\
\hline Weight, kg & $70.4 \pm 14.0$ & $78.3 \pm 12.2$ & $63.5 \pm 11.7$ & $<0.01$ \\
\hline Height, m & $1.71 \pm 0.01$ & $1.78 \pm 0.07$ & $1.64 \pm 0.07$ & $<0.01$ \\
\hline $\mathrm{BMI}, \mathrm{kg} / \mathrm{m}^{2}$ & $23.5(21.5-23.9)$ & $24.2(22.7-26.5)$ & $22.5(20.8-25.4)$ & 0.01 \\
\hline$W C, \mathrm{~cm}$ & $83.8 \pm 11.9$ & $89.1 \pm 10.3$ & $79.2 \pm 11.2$ & $<0.01$ \\
\hline $\mathrm{HC}, \mathrm{cm}$ & $101 \pm 9$ & $102 \pm 9$ & $100 \pm 10$ & 0.04 \\
\hline WHR & $0.83 \pm 0.08$ & $0.88 \pm 0.07$ & $0.79 \pm 0.08$ & $<0.01$ \\
\hline WHtR & $0.49 \pm 0.07$ & $0.50 \pm 0.06$ & $0.48 \pm 0.07$ & $<0.01$ \\
\hline \multicolumn{5}{|l|}{ Blood pressure, $\mathrm{mmHg}$} \\
\hline Systolic & $120 \pm 14$ & $124 \pm 11$ & $117 \pm 15$ & $<0.01$ \\
\hline Diastolic & $72 \pm 9$ & $74 \pm 9$ & $70 \pm 9$ & $<0.01$ \\
\hline Pulse pressure & $48 \pm 11$ & $50 \pm 10$ & $47 \pm 10$ & $<0.01$ \\
\hline \multicolumn{5}{|l|}{ Body composition measures } \\
\hline Body fat, $\%$ & $28.3 \pm 8.4$ & $23.7 \pm 7.2$ & $32.3 \pm 7.4$ & $<0.01$ \\
\hline Fat mass, kg & $19.0(14.3-25.0)$ & $17.8(12.9-24.9)$ & $19.3(15.5-25.2)$ & 0.01 \\
\hline Lean mass, kg & $48.4 \pm 10.5$ & $40.7 \pm 5.7$ & $57.2 \pm 7.4$ & $<0.01$ \\
\hline Trunk fat mass, kg & $10.4 \pm 5.0$ & $10.9 \pm 5.2$ & $10.0 \pm 4.9$ & 0.09 \\
\hline Abdominal VAT, $g$ & $393(178-811)$ & $691(367-1240)$ & $237(99-440)$ & $<0.01$ \\
\hline Android fat, \% & $30.5 \pm 12.1$ & $29.1 \pm 12.1$ & $31.8 \pm 11.8$ & 0.03 \\
\hline Gynoid fat, \% & $32.2 \pm 9.9$ & $24.9 \pm 7.0$ & $38.7 \pm 7.2$ & 0.01 \\
\hline $\mathrm{A} / \mathrm{G}$ fat ratio & $0.96 \pm 0.29$ & $1.13 \pm 0.28$ & $0.80 \pm 0.21$ & 0.01 \\
\hline \multicolumn{5}{|l|}{ Body Composition Indexes } \\
\hline $\mathrm{FMl}, \mathrm{kg} / \mathrm{m}^{2}$ & $7.05 \pm 2.93$ & $6.10 \pm 2.43$ & $7.88 \pm 3.09$ & 0.01 \\
\hline $\mathrm{LMI}, \mathrm{kg} / \mathrm{m}^{2}$ & $16.4 \pm 2.2$ & $18.0 \pm 1.7$ & $15.0 \pm 1.5$ & 0.01 \\
\hline VAl & $1.01 \pm 0.68$ & $1.03 \pm 0.71$ & $1.00 \pm 0.65$ & 0.66 \\
\hline \multicolumn{5}{|l|}{ Biochemistry } \\
\hline $\mathrm{TC}, \mathrm{mmol} / \mathrm{L}$ & $5.13 \pm 1.10$ & $5.05 \pm 1.18$ & $5.20 \pm 1.02$ & 0.10 \\
\hline $\mathrm{TAG}, \mathrm{mmol} / \mathrm{L}$ & $0.83(0.66-1.16)$ & $0.93(0.69-1.39)$ & $0.79(0.64-1.02)$ & 0.01 \\
\hline $\mathrm{HDL}-\mathrm{C}, \mathrm{mmol} / \mathrm{L}$ & $1.65 \pm 0.40$ & $1.51 \pm 0.40$ & $1.78 \pm 0.36$ & 0.01 \\
\hline $\mathrm{LDL}-\mathrm{C}, \mathrm{mmol} / \mathrm{L}$ & $3.03 \pm 0.93$ & $3.07 \pm 1.00$ & $2.99 \pm 0.86$ & 0.66 \\
\hline Non-HDL-C, mmol/L & $3.48 \pm 1.00$ & $3.55 \pm 1.07$ & $3.43 \pm 0.94$ & 0.34 \\
\hline TC:HDL ratio & $3.00(2.63-3.76)$ & $3.44(2.78-4.03)$ & $2.81(2.56-3.29)$ & 0.01 \\
\hline LDL-C:HDL-C ratio & $1.76(1.42-2.30)$ & $2.08(1.58-2.56)$ & $1.60(1.35-2.04)$ & 0.01 \\
\hline Glucose, mmol/L & $5.03 \pm 0.48$ & $5.13 \pm 0.51$ & $4.94 \pm 0.44$ & 0.01 \\
\hline Insulin, pmol/L & $26.4(17.3-39.9)$ & $27.1(16.9-42.5)$ & $26.3(18.2-37.7)$ & 0.69 \\
\hline HOMA-IR & $0.98(0.07-5.30)$ & $1.04(0.63-1.63)$ & $0.97(0.62-1.41)$ & 0.41 \\
\hline $\mathrm{NEFA}, \mu \mathrm{mol} / \mathrm{L}$ & $416(318-546)$ & $388(310-518)$ & $427(327-567)$ & 0.01 \\
\hline CRP, mg/L & $0.62(0.29-1.46)$ & $0.63(0.31-1.43)$ & $0.62(0.28-1.52)$ & 0.91 \\
\hline GGT, U/L & $16.9(14.0-22.7)$ & $20.5(16.2-27.5)$ & $15.3(13.2-19.0)$ & 0.01 \\
\hline Uric acid, $\mu \mathrm{mol} / \mathrm{L}$ & $280 \pm 68$ & $323 \pm 59$ & $242 \pm 51$ & 0.01 \\
\hline Adiponectin, $\mu \mathrm{g} / \mathrm{mL}$ & $5.11(2.48-9.07)$ & $4.19(2.22-6.02)$ & $6.70(2.93-11.38)$ & 0.01 \\
\hline 25-Hydroxyvitamin D, ng/mL & $23.9 \pm 11.3$ & $23.4 \pm 10.8$ & $24.3 \pm 11.7$ & 0.50 \\
\hline
\end{tabular}

A/G fat ratio android to gynoid fat ratio, BMI body mass index, CRP C-reactive protein, $F$ female, $F F M$ fat free mass, $F M I$ fat mass index, GGT gamma-glutamyl transferase, $H C$ hip circumference, HDL-C high density lipoprotein cholesterol, LDL-C low density lipoprotein cholesterol, LMI lean mass index, $M$ male, NEFA non-esterified fatty acids, TAG triacylglycerol, TC total cholesterol, VAT visceral adipose tissue, VAI visceral adiposity index, WC waist circumference, WHR waist to hip ratio, WHtR waist to height ratio

${ }^{a}$ Data were analyzed by independent $t$ tests and presented as mean \pm SD or median (interquartile range); $p \leq 0.05$ was considered significant

${ }^{b}$ Sample sizes differ as follows: Blood pressure $n=406$ (M:187/F:219); body composition measures $n=370$ (M:174/F:196); biochemistry $n=405$ (M:188/F:217); insulin and HOMA-IR $n=272$ (M:109/F:163); NEFA $n=362$ (M:168/F:194); CRP $n=403$ (M:188/F:215), GGT $n=330$ (M:135/F:195); UA, adiponectin and 25-hydroxyvitamin D, $\mathrm{n}=366(\mathrm{M}: 172 / \mathrm{F}: 194)$ 
Table 2 Dietary intake and physical activity levels of the study participants

\begin{tabular}{|c|c|c|c|c|}
\hline & All $(n=391)$ & Men $(n=179)$ & Women $(n=239)$ & $p$ value $^{\mathrm{a}}$ \\
\hline \multicolumn{5}{|l|}{ Dietary energy and macronutrient intake } \\
\hline Energy, MJ/day & $8.50 \pm 2.47$ & $9.62 \pm 2.51$ & $7.56 \pm 2.00$ & $<0.01$ \\
\hline Total Fat, \%TE & $36.5 \pm 8.6$ & $34.6 \pm 9.6$ & $36.4 \pm 7.8$ & 0.82 \\
\hline SFA, \%TE & $13.0 \pm 4.5$ & $13.3 \pm 5.2$ & $12.8 \pm 3.7$ & 0.36 \\
\hline MUFA, \%TE & $13.7 \pm 3.8$ & $13.6 \pm 4.1$ & $13.8 \pm 3.6$ & 0.70 \\
\hline PUFA, \%TE & $6.17 \pm 2.11$ & $6.09 \pm 2.33$ & $6.24 \pm 1.91$ & 0.51 \\
\hline n-6 PUFA, \%TE & $5.66 \pm 2.91$ & $5.33 \pm 3.03$ & $5.93 \pm 2.79$ & 0.04 \\
\hline n-3 PUFA, \%TE & $0.86 \pm 0.59$ & $0.82 \pm 0.49$ & $0.90 \pm 0.67$ & 0.17 \\
\hline Trans fat, \%TE & $0.49(0.34-0.68)$ & $0.50(0.35-0.72)$ & $0.49(0.33-0.63)$ & 0.05 \\
\hline Protein, \%TE & $17.1(14.8-20.2)$ & $17.0(14.5-20.4)$ & $17.4(15.0-19.7)$ & 0.58 \\
\hline Carbohydrate, \%TE & $45.8 \pm 10.9$ & $45.4 \pm 12.1$ & $46.1 \pm 9.8$ & 0.52 \\
\hline Total Sugars, \%TE & $18.7 \pm 6.6$ & $17.7 \pm 7.0$ & $19.6 \pm 6.0$ & 0.01 \\
\hline Dietary Fibre (AOAC), g/day & $24.3 \pm 8.8$ & $25.2 \pm 8.9$ & $23.5 \pm 8.6$ & 0.07 \\
\hline \multicolumn{5}{|l|}{ Physical activity level $\left.\right|^{b}$} \\
\hline Steps/day & $8953(6948-11,941)$ & $8500(6517-10,717)$ & $9288(7193-12,024)$ & 0.02 \\
\hline Energy expended (kcal/day) & $254(157-431)$ & $324(195-524)$ & $224(141-349)$ & $<0.01$ \\
\hline \multicolumn{5}{|l|}{ Percentage time per day spent } \\
\hline Sedentary & $69.8 \pm 7.3$ & $71.1 \pm 7.4$ & $68.9 \pm 7.1$ & 0.01 \\
\hline Performing light PA & $25.5 \pm 6.8$ & $24.3 \pm 6.6$ & $26.3 \pm 6.9$ & 0.01 \\
\hline Performing moderate to vigorous PA & $4.2(2.7-6.2)$ & $4.0(2.6-6.1)$ & $4.4(2.7-6.3)$ & 0.34 \\
\hline
\end{tabular}

AOAC Association of Official Analytical Chemist, MUFA monounsaturated fatty acids, PA physical activity, PUFA polyunsaturated fatty acids, SFA saturated fatty acids, $\% T E \%$ of total energy

${ }^{a}$ Differences between men and women were analyzed by independent $\mathrm{t}$ test and presented as mean $\pm \mathrm{SD}$ or median (interquartile range); $p \leq 0.05$ was considered significant

b Sample sizes differed as follows: Physical activity level $n=327$ (M:126/F:201) and steps/day n=309 (M:120/F:189)

between cardiometabolic disease risk markers with body composition measurements and dietary macronutrients in the whole group and in men and women separately (Spearman's Rho $\left(r_{s}\right)=0-0.3$ considered a weak correlation, $r_{s}=0.3-0.7$ moderate and $r_{s}=0.7-1.0$ strong). Stepwise multiple linear regression analysis was performed using P-in of 0.05 and P-out of 0.01 to establish the independent associations between LDL-C and abdominal VAT mass with the anthropometric measures, cardiometabolic disease risk markers and dietary macronutrients.

For further analysis, the study cohort with dietary data was stratified according to dietary SFA intake expressed as \%TE. Subjects in Q1 were selected to be within dietary recommendations for SFA $(\leq 10 \% \mathrm{TE})$. General linear model (ANCOVA) was performed to investigate the impact of increasing intakes of dietary SFAs on subject characteristics, adjusting for age and sex. Post-hoc analyses with a Bonferroni correction were used to compare differences between the SFA\%TE quartile groups. Results are presented as estimated marginal means \pm SE for normally distributed and as median (interquartile range) for non-normally distributed variables in Table 5 and $p \leq 0.05$ was considered significant.

\section{Results}

\section{Study participants}

A total of 438 healthy subjects were recruited, 29 of them dropped out between the screening and the main visit and 409 subjects (219 were women and 190 men) completed the study. The flow of participants in the study is shown in Fig. 1. The cohort had a mean age of $42 \pm 16$ years and median BMI of 23.5 (IQR 21.5-25.9) $\mathrm{kg} / \mathrm{m}^{2}$. The main characteristics of the BODYCON

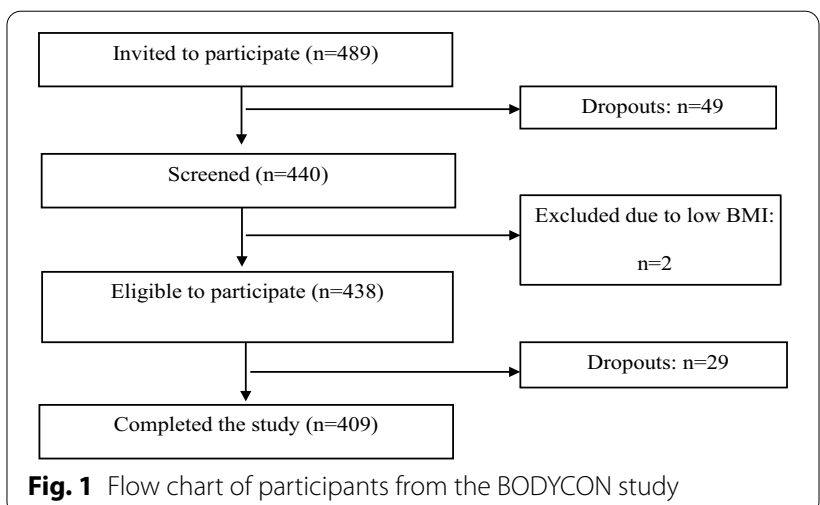


study participants are shown in Table 1. Men (47\%) and women (53\%) were approximately equally distributed and matched for age in the study population. Compared with women, men had greater BMI, body weight, WC, WHR, WHtR, SBP, and DBP ( $p<0.01$ each). Women had significantly higher body fat, android fat percentage, gynoid fat percentage and fat mass $(p \leq 0.03)$, whilst men had a higher lean body mass, abdominal VAT mass, and android:gynoid (A/G) percentage fat ratio ( $p<0.01$ each). Moreover, men had higher fasting serum TAG, glucose, GGT and UA concentrations and TC: HDL-C ratio $(p<0.01$ for all), while women had higher HDL-C, NEFA and adiponectin concentrations $(p \leq 0.01)$ (Table 1$)$.

The dietary intakes $(\mathrm{n}=391)$ and physical activity $(n=327)$ levels of the study participants are shown in Table 2. Within the cohort, 2 subjects were identified as under-reporters and 3 as over-reporters, with 13 further subjects excluded due to completion of $<3$ days of dietary intake $(n=1)$ or did not provide a diet diary $(n=12)$. For the mean dietary intakes, men reported greater energy intakes $(p<0.01)$, but only trans-fat (\%TE) intake was higher in men in terms of dietary macronutrients compared to women $(p=0.05)$, while women reported higher total sugar (\%TE) and n-6 PUFA (\%TE) intakes compared to men $(p \leq 0.04)$. Regarding physical activity levels, 82 subjects were excluded according to inclusion criteria for the physical activity analysis. Compared with men, women had higher daily step counts and spent a greater percentage of time during the day performing light physical activity $(p \leq 0.02)$. On average, men expended significantly more energy per day (approximately $100 \mathrm{kcal} /$ day) performing physical activity compared with women $(p<0.01)$. The percentage of time spent performing moderate to vigorous physical activity daily was not different between the sexes $(p=0.34)$ (Table 2).

\section{Association between body composition, cardiometabolic disease risk markers and dietary macronutrients}

Correlations between body composition measurements, CVD risk markers and dietary macronutrients in the whole group are shown in Table 3 and Additional file 1: Table 1 and according to sex in Additional file 1: Tables S2 and S3. In the whole group body fat mass was found to have weak positive correlations with SBP and DBP, while abdominal VAT mass and A/G fat ratio had moderate positive correlations with both SBP and DBP $(p<0.01)$. In addition, inverse moderate correlations were evident between HDL-C and several adiposity measurements, including abdominal VAT mass $(p<0.01)$. In contrast, moderate positive correlations were found between TAG, non-HDL-C, TC: HDL-C ratio and LDL-C: HDL-C ratio with abdominal VAT mass, android fat mass, android fat percentage and A/G fat ratio $(p<0.01$ for each). There were also weak positive correlations between LDL-C with SFA (\%TE) and trans-fat (\%TE) $(p<0.01$ for each). Weak correlations were found between dietary macronutrients and cardiometabolic disease risk markers, with SFA (\%TE) intake positively associated with TC, LDL-C, non-HDL-C and NEFA $(p \leq 0.05)$, whereas carbohydrate (\%TE) intake was negatively correlated with LDL-C $(p<0.01)$.

For abdominal VAT mass, moderate positive correlations were found with insulin, HOMA-IR, glucose, CRP and uric acid, while there were weak, negative correlations with adiponectin and 25-hydroxyvitamin D levels $(p \leq 0.05)$. Regarding the association between diet and body composition, we observed weak correlations. n-6 PUFA (\%TE) intake was negatively correlated with abdominal VAT mass, while trans-fat (\%TE) intake was positively correlated $(p<0.01)$ (Table 3$)$.

After stratifying the group according to sex, a few sex-specific associations were observed. Body fat mass and abdominal VAT mass were found to have weak to moderate positive correlations with both SBP and DBP in women, while only with DBP in men $(p<0.01)$. In addition, inverse moderate correlations were evident between HDL-C and several adiposity measurements, including abdominal VAT mass in men $(p<0.01)$, while there were weak inverse correlations between HDL-C and percentage body fat, fat mass and android fat percentage in women $(p<0.05)$. Abdominal VAT mass was negatively correlated with n-6 PUFA (\%TE) in men $(p<0.01)$, whilst in women there was a weak inverse correlation with carbohydrate $(\% \mathrm{TE})$ intake $(p<0.05)$ (Additional file 1: S3).

\section{Stepwise multivariate regression analysis}

The standardized regression coefficients, adjusted $r^{2}$ and $p$ values for the stepwise multivariate regression analysis are shown in Table 4. Only SFA (\%TE) intake, abdominal VAT mass, total fat (\%TE) and carbohydrate (\%TE) intakes were found to be independently associated with fasting LDL-C, explaining $18.3 \%$ of the variability in this established CVD risk marker. Of these variables, $9 \%$ of this variability was explained by SFA (\%TE) intake and $7 \%$ by abdominal VAT mass (Table 4 ).

The TC: HDL-C ratio, DBP, GGT, HOMA-IR, sex, age, HDL-C and uric acid were independently associated with abdominal VAT mass and, together, these variables explained $64 \%$ of the variability in abdominal VAT mass. This analysis showed that TC: HDL-C ratio alone explained $33 \%$ of the variability in the mass of this fat depot. 
Table 3 Spearman's correlation coefficients $\left(r_{s}\right)$ for the relationship between DXA body composition measurements, with CVD risk factors and dietary macronutrients

\begin{tabular}{|c|c|c|c|c|c|c|c|c|}
\hline & Body fat, $\%$ & Fat mass, kg & Lean mass, kg & VAT, $g$ & Android fat, kg & Android fat $\%$ & Gynoid fat \% & $A / G$ \\
\hline \multicolumn{9}{|l|}{ Blood pressure, mmHg } \\
\hline Systolic & -0.03 & $0.15^{* *}$ & $0.33^{* *}$ & $0.40^{* *}$ & $0.25^{* *}$ & $0.14^{* *}$ & $-0.15^{* *}$ & $0.33^{* *}$ \\
\hline Diastolic & $0.13^{*}$ & $0.29^{* *}$ & $0.21^{* *}$ & $0.44^{* *}$ & $0.37^{* *}$ & $0.31^{* *}$ & -0.003 & $0.39^{* *}$ \\
\hline Pulse pressure & $-0.14^{* *}$ & -0.02 & $0.25^{* *}$ & $0.16^{* *}$ & 0.03 & -0.05 & $-0.19^{* *}$ & $0.11^{*}$ \\
\hline \multicolumn{9}{|l|}{ Biochemistry } \\
\hline $\mathrm{TC}, \mathrm{mmol} / \mathrm{L}$ & $0.19^{* *}$ & $0.17^{* *}$ & $-0.11^{*}$ & $0.25^{* *}$ & $0.23^{* *}$ & $0.23^{* *}$ & $0.13^{*}$ & $0.20^{* *}$ \\
\hline TAG, mmol/L & $0.21^{* *}$ & $0.35^{* *}$ & $0.18^{* *}$ & $0.46^{* *}$ & $0.42^{* *}$ & $0.38^{* *}$ & 0.06 & $0.43^{* *}$ \\
\hline $\mathrm{HDL}-\mathrm{C}, \mathrm{mmol} / \mathrm{L}$ & -0.02 & $-0.23^{* *}$ & $-0.35^{* *}$ & $-0.35^{* *}$ & $-0.29^{* *}$ & $-0.20^{* *}$ & $0.11^{*}$ & $-0.34^{* *}$ \\
\hline LDL-C, mmol/L & $0.17^{* *}$ & $0.20^{* *}$ & -0.02 & $0.32^{* *}$ & $0.27^{* *}$ & $0.25^{* *}$ & 0.09 & $0.26^{* *}$ \\
\hline Non-HDL-C, mmol/L & $0.22^{* *}$ & $0.27^{* *}$ & 0.02 & $0.41^{* *}$ & $0.36^{* *}$ & $0.33^{* *}$ & 0.10 & $0.35^{* *}$ \\
\hline TC: HDL ratio & $0.18^{* *}$ & $0.37^{* *}$ & $0.24^{* *}$ & $0.55^{* *}$ & $0.47^{* *}$ & $0.39^{* *}$ & -0.001 & $0.50^{* *}$ \\
\hline LDL-C: HDL-C ratio & $0.16^{* *}$ & $0.33^{* *}$ & $0.23^{* *}$ & $0.51^{* *}$ & $0.43^{* *}$ & $0.35^{* *}$ & -0.01 & $0.46^{* *}$ \\
\hline NEFA, $\mu \mathrm{mol} / \mathrm{L}$ & $0.20^{* *}$ & $0.12^{*}$ & $-0.17^{* *}$ & 0.04 & $0.10^{*}$ & $0.15^{* *}$ & $0.20^{* *}$ & -0.02 \\
\hline Glucose, $\mathrm{mmol} / \mathrm{L}$ & 0.07 & $0.23^{* *}$ & $0.22^{* *}$ & $0.41^{* *}$ & $0.31^{* *}$ & $0.23^{* *}$ & -0.05 & $0.35^{* *}$ \\
\hline Insulin, pmol/L & $0.35^{* *}$ & $0.41^{* *}$ & 0.02 & $0.34^{* *}$ & $0.41^{* *}$ & $0.42^{* *}$ & $0.24^{* *}$ & $0.32^{* *}$ \\
\hline HOMA-IR & $0.34^{* *}$ & $0.42^{* *}$ & 0.06 & $0.38^{* *}$ & $0.43^{* *}$ & $0.42^{* *}$ & $0.21^{* *}$ & $0.34^{* *}$ \\
\hline $\mathrm{CRP}, \mathrm{mg} / \mathrm{L}$ & $0.36^{* *}$ & $0.41^{* *}$ & 0.001 & $0.29^{* *}$ & $0.39^{* *}$ & $0.41^{* *}$ & $0.27^{* *}$ & $0.27^{* *}$ \\
\hline $\mathrm{GGT}, \mathrm{U} / \mathrm{L}$ & -0.09 & $0.11^{*}$ & $0.35^{* *}$ & $0.37^{* *}$ & $0.22^{* *}$ & 0.11 & $-0.22^{* *}$ & $0.36^{* *}$ \\
\hline Uric acid, $\mu \mathrm{mol} / \mathrm{L}$ & $-0.16^{* *}$ & $0.13^{*}$ & $0.53^{* *}$ & $0.43^{* *}$ & $0.29^{* *}$ & $0.13^{*}$ & $-0.35^{* *}$ & $0.51^{* *}$ \\
\hline Adiponectin, $\mu \mathrm{g} / \mathrm{mL}$ & $0.18^{* *}$ & 0.02 & $-0.25^{* *}$ & $-0.14^{* *}$ & -0.05 & 0.02 & $0.25^{* *}$ & -0.21 \\
\hline Total 25(OH)D, ng/mL & $-0.16^{* *}$ & $-0.14^{* *}$ & 0.05 & $-0.12^{*}$ & -0.08 & $-0.11^{*}$ & $-0.15^{* *}$ & $-0.14^{* *}$ \\
\hline \multicolumn{9}{|l|}{ Dietary intake } \\
\hline Total fat, \%TE & 0.02 & 0.01 & -0.03 & 0.01 & 0.01 & 0.01 & 0.02 & 0.01 \\
\hline SFA \%TE & 0.04 & 0.06 & 0.01 & 0.08 & 0.06 & 0.04 & 0.03 & 0.04 \\
\hline MUFA, \%TE & 0.01 & -0.01 & -0.05 & -0.02 & -0.01 & -0.004 & 0.02 & -0.01 \\
\hline PUFA, \%TE & -0.07 & $-0.15^{* *}$ & -0.09 & $-0.13^{*}$ & $-0.16^{* *}$ & $-0.13^{*}$ & -0.04 & $-0.14^{* *}$ \\
\hline n-6 PUFA, \%TE & -0.08 & $-0.16^{* *}$ & $-0.11^{*}$ & $-0.17^{* *}$ & $-0.19^{* *}$ & $-0.15^{* *}$ & -0.02 & $-0.18^{* *}$ \\
\hline n-3 PUFA, \%TE & -0.004 & -0.05 & -0.08 & 0.01 & -0.04 & -0.01 & 0.02 & -0.03 \\
\hline Trans fat, \%TE & 0.07 & $0.11^{*}$ & 0.07 & $0.16^{* *}$ & $0.13^{* *}$ & $0.11^{*}$ & 0.04 & $0.12^{*}$ \\
\hline Protein, \%TE & -0.03 & -0.003 & 0.07 & -0.04 & -0.02 & -0.05 & -0.03 & -0.07 \\
\hline Carbohydrate, \%TE & 0.02 & 0.003 & -0.05 & -0.03 & 0.001 & 0.02 & 0.03 & 0.01 \\
\hline Fibre (AOAC), g/day & $-0.22^{* *}$ & $-0.14^{* *}$ & $0.21^{* *}$ & -0.06 & $-0.13^{*}$ & $-0.21^{* *}$ & $-0.21^{* *}$ & -0.07 \\
\hline Total Sugars, \%TE & 0.03 & -0.01 & $-0.11^{*}$ & $-0.14^{* *}$ & -0.04 & -0.04 & 0.07 & $-0.11^{*}$ \\
\hline
\end{tabular}

Data analysed by Spearman's correlations

$A O A C$ association of analytical chemists, $A / G$ android to gynoid ratio, $C R P C$-reactive protein, GGT gamma-glutamyl transferase, HDL-C high density lipoprotein cholesterol, HOMA-IR homeostatic model assessment for insulin resistance, LDL-C low density lipoprotein cholesterol, MUFA monounsaturated fatty acids, NEFA nonesterified fatty acids, PUFA polyunsaturated fatty acids, SFA saturated fatty acids, TC total cholesterol, TAG triacylglycerol, \%TE \% of total energy, VAT abdominal visceral adipose tissue, total 25(OH)D: 25-hydroxyvitamin D

*Significant differences at the 0.05 level

**Significant differences at the 0.01 level

\section{Subject characteristics according to quartiles of dietary SFA (\%TE) intake}

There were no significant differences in mean body weight $(p=0.10)$ or BMI $(p=0.20)$ across quartiles $(\mathrm{Q})$ of increasing \%TE from SFA (Table 5). However, android lean mass was found to be 7\% higher in Q3 compared with Q1 $(p=0.02)$. Other anthropometric measures were not different across the quartiles of SFA\%TE intake.
Significant differences in several cardiometabolic disease risk markers were also evident across increasing quartiles of SFA (\%TE) intake. SBP and pulse pressure were higher in $\mathrm{Q} 4$ compared to Q3 $(p \leq 0.01)$. TC, LDL-C and non-HDL-C levels were $9 \%, 12 \%$ and $10 \%$ higher in Q4 than Q2, respectively $(p \leq 0.05)$. Regarding dietary intakes, subjects in Q4 reported higher total fat, MUFA and trans-fat (\%TE) than other quartiles $(p<0.01$ each) 
Table 4 Stepwise multivariate linear regression analysis exploring the relation between dietary macronutrients, body composition and biochemical variables with LDL-C and abdominal VAT

\begin{tabular}{|c|c|c|c|c|}
\hline Dependent variable & Independent variable & Standardized coefficient & Adjusted $r^{b}$ & $p$ value \\
\hline \multirow[t]{4}{*}{ LDL-Ca } & SFA \%TE & 0.297 & 0.085 & $<0.01$ \\
\hline & and Abdominal VAT & 0.277 & 0.160 & $<0.01$ \\
\hline & and Carbohydrate \% TE & -0.157 & 0.172 & 0.013 \\
\hline & and Fat \%TE & -0.261 & 0.183 & 0.017 \\
\hline \multirow[t]{8}{*}{ Abdominal VAT ${ }^{b}$} & TC: HDL-C & 0.572 & 0.325 & $<0.01$ \\
\hline & and DBP & 0.314 & 0.415 & $<0.01$ \\
\hline & and GGT & 0.253 & 0.475 & $<0.01$ \\
\hline & and HOMA-IR & 0.240 & 0.518 & $<0.01$ \\
\hline & and Sex (female) & -0.237 & 0.565 & $<0.01$ \\
\hline & and Age & 0.222 & 0.606 & $<0.01$ \\
\hline & and $\mathrm{HDL}-\mathrm{C}$ & -0.202 & 0.625 & 0.001 \\
\hline & and Uric acid & 0.157 & 0.636 & 0.005 \\
\hline
\end{tabular}

GGT gamma-glutamyl transferase, HDL-C high density lipoprotein cholesterol, HOMA-IR homeostatic model assessment for insulin resistance, $L D L-C$ low-density lipoprotein cholesterol, SFA saturated fatty acid, TC total cholesterol, \%TE \% of total energy, VAT visceral adipose tissue

${ }^{a}$ Variables included in the analysis: BMI, body fat \%, fat mass, abdominal VAT, android fat mass, android fat \%, A/G fat ratio, WC, HC, WHR, WHtR, fat \%TE, SFA \%TE, trans-fat \% TE, CHO \%TE

${ }^{b}$ Variables included in the analysis: age, sex, TC, TAG, HDL-C, LDL-C, non-HDL-C, TC: HDL-C, LDL-C: HDL-C, glucose, 25(OH)D, CRP, GGT, UA, adiponectin, insulin, HOMA-IR, SBP, DBP, PP, PUFA \%TE, n-6 PUFA \%TE, trans-fat \%TE, total sugars \%TE

and lower n-6 PUFA (\%TE) intake than Q2 $(p<0.01)$. Carbohydrate (\%TE) and fiber (g/day) intakes were lowest in Q4 compared to other quartiles $(p<0.01$ each) (Table 5).

\section{Discussion}

The present study investigated the associations between dietary SFA intake, cardiometabolic disease risk markers and body composition to determine whether body fat distribution contributed to the relationship between SFA and LDL-C in a group of healthy adults. Although our study does not establish cause and effect relationships due to its cross-sectional nature, we observed interesting and novel associations. In particular, dietary SFA, total fat and carbohydrate intakes and abdominal VAT mass were independently associated with LDL-C and found to explain $18.3 \%$ of the variability. However, SFA intake was not related to abdominal VAT mass. Furthermore, stratification according to quartiles of dietary SFA intake did not reveal dose-dependent relationships with LDL-C, TC, non-HDL-C, blood pressure or android lean mass.

The replacement of dietary SFA with unsaturated fatty acids (n-6 PUFA and MUFA) is associated with beneficial effects on the fasting blood lipid profiles [37]. In the PURE cross-sectional study, which included 104 486 men and women aged 30-70 years from 18 countries, dietary SFA intake was positively related with LDL-C and replacing 5\%TE of dietary SFA with PUFA and MUFA was associated with lower LDL-C concentrations (between 0.02 and $0.18 \mathrm{mmol} / \mathrm{L}$ ) using a multivariable nutrient density model [38]. In agreement with previous studies, we also observed an independent positive association between LDL-C and dietary SFA, with dietary SFAs explaining 9\% of the variability in LDL-C response between individuals. However, after stratifying data by SFA intake, we did not observe a linear relationship between increasing SFA intakes and LDL-C, with differences only evident in TC, LDL-C and non-HDL-C concentrations between Q2 and Q4. The lack of a dose-dependent relationship between SFA intake and LDL-C may reflect the use of age and sex as co-variates in the ANCOVA analysis, which are both important non-modifiable determinants of LDL-C concentrations [39, 40]. Furthermore, the association of dietary SFA with CVD risk has been proposed to be dependent on the food source and the type of individual SFA rather than the amount of the SFA. For example, although high in SFA, dairy have been reported to have neutral or positive effects on CVD risk markers [41], whereas palmitic acid has been reported to be more atherogenic than stearic acid [42] Therefore, determining total dietary SFA intake in the current study may have influenced the strength of the relationship with fasting LDL-C due to the differences in frequency of dairy product and/or individual SFA consumption within the quartile groups [41]. Interestingly, n-6 PUFA intake was considerably higher in Q2 compared to Q4, which may have also influenced blood cholesterol levels. Furthermore, high intakes of plant-based MUFA are associated with lower LDL-C 
Table 5 Participant's characteristics according to quartiles of dietary saturated fatty acid (\%TE) intake

\begin{tabular}{|c|c|c|c|c|c|}
\hline Characteristics $^{2}$ & $\begin{array}{l}\text { Q1 }(n=78) \\
(1.9-10.0 \% T E)\end{array}$ & $\begin{array}{l}Q 2(n=101) \\
(10.1-11.9 \% \text { TE })\end{array}$ & $\begin{array}{l}\text { Q3 }(n=109) \\
(12.0-14.8 \% T E)\end{array}$ & $\begin{array}{l}\mathrm{Q} 4(n=103) \\
(14.9-38.7 \% \mathrm{TE})\end{array}$ & $p$ value $^{1}$ \\
\hline Weight, kg & $68.4 \pm 1.3$ & $69.5 \pm 1.2$ & $72.5 \pm 1.1$ & $69.9 \pm 1.2$ & 0.10 \\
\hline $\mathrm{BMI}, \mathrm{kg} / \mathrm{m}^{2}$ & $23.0(20.8-25.4)$ & $23.2(21.4-25.5)$ & $24.1(22.0-27.0)$ & $23.6(21.5-25.7)$ & 0.20 \\
\hline$W C, c m$ & $82.5 \pm 1.1$ & $83.5 \pm 1.0$ & $85.4 \pm 1.0$ & $83.3 \pm 1.0$ & 0.21 \\
\hline $\mathrm{HC}, \mathrm{cm}$ & $100 \pm 1$ & $100 \pm 1$ & $103 \pm 1$ & $101 \pm 1$ & 0.06 \\
\hline WHR & $0.83 \pm 0.01$ & $0.83 \pm 0.01$ & $0.83 \pm 0.01$ & $0.83 \pm 0.01$ & 0.99 \\
\hline WHtR & $0.49 \pm 0.01$ & $0.49 \pm 0.01$ & $0.50 \pm 0.01$ & $0.49 \pm 0.01$ & 0.42 \\
\hline \multicolumn{6}{|l|}{ Blood pressure, $\mathrm{mmHg}$} \\
\hline SBP & $120 \pm 2^{\mathrm{ab}}$ & $121 \pm 1^{\mathrm{ab}}$ & $117 \pm 1^{b}$ & $123 \pm 2^{\mathrm{a}}$ & 0.01 \\
\hline DBP & $71 \pm 1$ & $72 \pm 1$ & $71 \pm 1$ & $72 \pm 1$ & 0.83 \\
\hline Pulse pressure & $48 \pm 1^{\mathrm{ab}}$ & $49 \pm 1^{\mathrm{a}}$ & $45 \pm 1^{b}$ & $50 \pm 1^{\mathrm{a}}$ & $<0.01$ \\
\hline \multicolumn{6}{|l|}{ Body composition measures } \\
\hline Body fat, $\%$ & $28.4 \pm 0.8$ & $28.3 \pm 0.7$ & $29.1 \pm 0.7$ & $27.6 \pm 0.7$ & 0.53 \\
\hline Android fat, $\%$ & $30.5 \pm 1.4$ & $30.8 \pm 1.2$ & $31.6 \pm 1.2$ & $29.6 \pm 1.2$ & 0.68 \\
\hline Gynoid fat, \% & $32.5 \pm 0.8$ & $32.2 \pm 0.7$ & $33.2 \pm 0.7$ & $31.6 \pm 0.7$ & 0.47 \\
\hline A/G fat ratio & $0.94 \pm 0.03$ & $0.96 \pm 0.02$ & $0.97 \pm 0.02$ & $0.94 \pm 0.02$ & 0.79 \\
\hline Fat mass, kg & $17.7(12.3-25.3)$ & $17.9(14.5-25.0)$ & $20.2(16.1-25.4)$ & $19.0(14.3-24.0)$ & 0.21 \\
\hline Lean mass, kg & $46.8 \pm 0.8$ & $48.0 \pm 0.7$ & $49.2 \pm 0.7$ & $48.3 \pm 0.7$ & 0.12 \\
\hline Android fat mass, kg & $1.63 \pm 0.12$ & $1.57 \pm 0.10$ & $1.72 \pm 0.10$ & $1.54 \pm 0.10$ & 0.58 \\
\hline Android lean mass, kg & $3.19 \pm 0.06^{\mathrm{a}}$ & $3.30 \pm 0.05^{\mathrm{ab}}$ & $3.42 \pm 0.05^{b}$ & $3.28 \pm 0.05^{a b}$ & 0.02 \\
\hline Abdominal VAT, g & $562 \pm 56$ & $582 \pm 50$ & $651 \pm 48$ & $562 \pm 49$ & 0.53 \\
\hline \multicolumn{6}{|l|}{ Indexes } \\
\hline VAI & $1.05 \pm 0.08$ & $1.02 \pm 0.07$ & $1.06 \pm 0.07$ & $0.94 \pm 0.07$ & 0.61 \\
\hline \multicolumn{6}{|l|}{ Biochemistry } \\
\hline $\mathrm{TC}, \mathrm{mmol} / \mathrm{L}$ & $5.17 \pm 0.11^{\mathrm{ab}}$ & $4.91 \pm 0.10^{\mathrm{a}}$ & $5.10 \pm 0.09^{\mathrm{ab}}$ & $5.39 \pm 0.09^{b}$ & 0.01 \\
\hline $\mathrm{TAG}, \mathrm{mmol} / \mathrm{L}$ & $0.84(0.67-1.18)$ & $0.82(0.65-1.07)$ & $0.84(0.66-1.28)$ & $0.84(0.67-1.11)$ & 0.59 \\
\hline $\mathrm{HDL}-\mathrm{C}, \mathrm{mmol} / \mathrm{L}$ & $1.67 \pm 0.04$ & $1.61 \pm 0.04$ & $1.61 \pm 0.04$ & $1.73 \pm 0.04$ & 0.05 \\
\hline LDL-C, mmol/L & $3.00 \pm 1.00^{\mathrm{ab}}$ & $2.87 \pm 0.08^{\mathrm{a}}$ & $3.04 \pm 0.08^{\mathrm{ab}}$ & $3.23 \pm 0.08^{b}$ & 0.03 \\
\hline Non-HDL, mmol/L & $3.50 \pm 0.10^{\mathrm{ab}}$ & $3.31 \pm 0.09^{\mathrm{a}}$ & $3.49 \pm 0.09^{\mathrm{ab}}$ & $3.66 \pm 0.09^{b}$ & 0.05 \\
\hline TC: HDL-C & $2.95(2.65-3.71)$ & $2.94(2.61-3.44)$ & $3.03(2.61-3.81)$ & $3.15(2.61-3.83)$ & 0.61 \\
\hline LDL-C: HDL-C & $1.69(1.43-2.23)$ & $1.71(1.42-2.13)$ & $1.82(1.41-2.42)$ & $1.90(1.41-2.40)$ & 0.50 \\
\hline NEFA, $\mu \mathrm{mol} / \mathrm{L}$ & $390(327-534)$ & $403(294-500)$ & $441(310-560)$ & $456(340-598)$ & 0.36 \\
\hline Glucose, mmol/L & $4.98 \pm 0.05$ & $5.08 \pm 0.04$ & $5.01 \pm 0.04$ & $5.02 \pm 0.04$ & 0.49 \\
\hline CRP, mg/L & $0.49(0.25-1.29)$ & $0.59(0.27-1.37)$ & $0.66(0.38-1.54)$ & $0.66(0.29-1.69)$ & 0.79 \\
\hline Uric acid, $\mu \mathrm{mol} / \mathrm{L}$ & $270 \pm 7$ & $275 \pm 6$ & $289 \pm 6$ & $282 \pm 6$ & 0.14 \\
\hline Adiponectin, $\mu \mathrm{g} / \mathrm{mL}$ & $6.03(2.50-10.62)^{\mathrm{a}}$ & $4.93(2.39-7.99)^{a b}$ & $4.09(1.99-8.12)^{b}$ & $5.45(3.11-9.87)^{a b}$ & 0.03 \\
\hline Total 25-Hydroxyvitamin D, ng/mL & $22.3 \pm 1.3$ & $24.3 \pm 1.2$ & $24.3 \pm 1.1$ & $24.2 \pm 1.2$ & 0.63 \\
\hline Insulin, pmol/L & $26.0(20.3-37.5)$ & $26.5(17.3-38.6)$ & $22.4(15.9-40.1)$ & $28.7(18.3-42.7)$ & 0.61 \\
\hline HOMA-IR & $1.00(0.68-1.44)$ & $1.00(0.65-1.53)$ & $0.85(0.58-1.44)$ & $1.15(0.66-1.63)$ & 0.60 \\
\hline \multicolumn{6}{|l|}{ Dietary intake } \\
\hline Energy, kcal/day & $1906 \pm 61$ & $2045 \pm 54$ & $2055 \pm 51$ & $2092 \pm 53$ & 0.13 \\
\hline Energy, MJ/day & $7.98 \pm 0.26$ & $8.56 \pm 0.22$ & $8.60 \pm 0.22$ & $8.75 \pm 0.22$ & 0.13 \\
\hline Total fat, \%TE & $28.2 \pm 0.7^{a}$ & $34.3 \pm 0.6^{b}$ & $36.5 \pm 0.6^{b}$ & $44.9 \pm 0.6^{c}$ & $<0.01$ \\
\hline MUFA, \%TE & $11.0 \pm 0.4^{b}$ & $13.3 \pm 0.3^{\mathrm{a}}$ & $13.8 \pm 0.3^{\mathrm{a}}$ & $16.1 \pm 0.3^{c}$ & $<0.01$ \\
\hline PUFA, \%TE & $5.97 \pm 0.24$ & $6.39 \pm 0.21$ & $6.04 \pm 0.20$ & $6.24 \pm 0.21$ & 0.52 \\
\hline n-6 PUFA, \%TE & $5.64 \pm 0.33^{\mathrm{ab}}$ & $6.48 \pm 0.29^{\mathrm{a}}$ & $5.53 \pm 0.28^{\mathrm{ab}}$ & $5.00 \pm 0.28^{b}$ & $<0.01$ \\
\hline n-3 PUFA, \%TE & $0.84 \pm 0.07^{a b}$ & $0.87 \pm 0.06^{\mathrm{ab}}$ & $0.76 \pm 0.06^{\mathrm{a}}$ & $0.98 \pm 0.06^{b}$ & 0.06 \\
\hline PUFA/SFA & $0.81 \pm 0.03^{\mathrm{a}}$ & $0.58 \pm 0.03^{b}$ & $0.46 \pm 0.03^{c}$ & $0.34 \pm 0.03^{d}$ & $<0.01$ \\
\hline MUFA/SFA & $1.44 \pm 0.04^{\mathrm{a}}$ & $1.21 \pm 0.03^{b}$ & $1.04 \pm 0.03^{c}$ & $0.89 \pm 0.03^{d}$ & $<0.01$ \\
\hline
\end{tabular}


Table 5 (continued)

\begin{tabular}{|c|c|c|c|c|c|}
\hline Characteristics $^{2}$ & $\begin{array}{l}\text { Q1 }(n=78) \\
(1.9-10.0 \% T E)\end{array}$ & $\begin{array}{l}Q 2(n=101) \\
(10.1-11.9 \% \text { TE })\end{array}$ & $\begin{array}{l}\text { Q3 }(n=109) \\
(12.0-14.8 \% T E)\end{array}$ & $\begin{array}{l}\mathrm{Q} 4(\mathrm{n}=103) \\
(14.9-38.7 \% \mathrm{TE})\end{array}$ & $p$ value $^{1}$ \\
\hline Trans fat, \%TE & $0.30(0.23-0.40)^{\mathrm{a}}$ & $0.41(0.31-0.54)^{b}$ & $0.53(0.43-0.68)^{c}$ & $0.76(0.56-0.96)^{d}$ & $<0.01$ \\
\hline Protein, \%TE & $17.6(14.7-20.5)$ & $18.1(15.7-20.9)$ & $16.5(14.5-19.2)$ & $16.5(14.5-19.8)$ & 0.44 \\
\hline Carbohydrate, \%TE & $54.1 \pm 1.1^{\mathrm{b}}$ & $47.3 \pm 0.9^{\mathrm{a}}$ & $46.1 \pm 0.9^{\mathrm{a}}$ & $37.7 \pm 0.9^{c}$ & $<0.01$ \\
\hline Fiber (AOAC), g/day & $27.3 \pm 1.0^{\mathrm{a}}$ & $26.3 \pm 0.9^{\mathrm{ab}}$ & $23.8 \pm 0.8^{b}$ & $20.5 \pm 0.8^{c}$ & $<0.01$ \\
\hline Total Sugars, \%TE & $19.6 \pm 0.7$ & $18.9 \pm 0.6$ & $19.4 \pm 0.6$ & $17.1 \pm 0.6$ & 0.03 \\
\hline \multicolumn{6}{|l|}{ Physical activity level } \\
\hline Steps/day & $9786(7583-12,573)$ & $9153(6883-11,523)$ & $8937(6876-11,973)$ & $8177(6715-11,206)$ & 0.25 \\
\hline Energy expended (kcal/day) & $296(164-525)$ & $265(162-450)$ & $249(146-385)$ & $231(144-330)$ & 0.16 \\
\hline \multicolumn{6}{|l|}{ Percentage time spent per day } \\
\hline Sedentary & $70.0 \pm 0.9$ & $69.7 \pm 0.8$ & $69.8 \pm 0.8$ & $70.1 \pm 0.8$ & 0.98 \\
\hline Performing light PA & $25.0 \pm 0.8$ & $25.7 \pm 0.7$ & $25.6 \pm 0.7$ & $25.4 \pm 0.7$ & 0.89 \\
\hline Performing moderate to vigorous PA & $4.6(2.9-6.8)$ & $4.2(3.1-6.4)$ & $4.0(2.5-6.6)$ & $4.0(2.5-5.6)$ & 0.64 \\
\hline
\end{tabular}

AOAC Association of Official Analytical Chemist, CRP C-reactive protein, $D B P$ diastolic blood pressure, $H C$ hip circumference, $H D L-C$ high density lipoprotein cholesterol LDL-C low density lipoprotein cholesterol, MUFA monounsaturated fatty acids, NEFA non-esterified fatty acids, PA physical activity, PUFA polyunsaturated fatty acids, SFA saturated fatty acids, SBP systolic blood pressure, TC total cholesterol, TAG triacylglycerol, UA uric acid, VAl visceral adiposity index, WC waist circumference, WHR waist to hip ratio, WHtR waist to height ratio

${ }^{1}$ Data were analysed by ANCOVA with age and sex as covariates and presented as estimated mariginal means $\pm \mathrm{SE}$ or median (interquartile range); $p \leq 0.05$ considered significant

${ }^{2}$ Sample sizes differ as follows: Blood pressure, $\mathrm{Q} 1 \mathrm{n}=77, \mathrm{Q} 2 \mathrm{n}=101, \mathrm{Q} 3 \mathrm{n}=107, \mathrm{Q} 4 \mathrm{n}=103$; body composition measures, $\mathrm{Q} 1 \mathrm{n}=72, \mathrm{Q} 2 \mathrm{n}=91, \mathrm{Q} 3 \mathrm{n}=98, \mathrm{Q} 4 \mathrm{n}=94$; VAl, Q1 $n=78, Q 2 n=99, Q 3 n=103, Q 4 n=103$; biochemistry, Q1 $n=78, Q 2 n=100, Q 3 n=106, Q 4 n=103 ; N E F A, Q 1 n=72, Q 2 n=89, Q 3 n=92, Q 4 n=94 ; C R P$, $\mathrm{Q} 1 \mathrm{n}=77, \mathrm{Q} 2 \mathrm{n}=99, \mathrm{Q} 3 \mathrm{n}=106, \mathrm{Q} 4 \mathrm{n}=103$; UA, adiponectin and 25-hydroxyvitamin $\mathrm{D}, \mathrm{Q} 1 \mathrm{n}=72, \mathrm{Q} 2 \mathrm{n}=90, \mathrm{Q} 3 \mathrm{n}=95, \mathrm{Q} 4 \mathrm{n}=94$; insulin and HOMA-IR, Q1 $\mathrm{n}=52$, Q2 $n=71, Q 3 n=67, Q 4 n=69$; dietary intake, Q1 $n=78, Q 2 n=101, Q 3 n=109, Q 4 n=103$; physical activity, Q1 $n=69, Q 2 n=80, Q 3 n=84, Q 4 n=85 ;$ steps/day, Q1 $n=66, Q 2 n=77, Q 3 n=77, Q 4 n=81$

concentrations [43] and in this cohort those with the highest SFA intakes also had higher total fat and MUFA intakes. However, this would not necessarily represent a higher intake of plant-based MUFA-rich foods and oils, as animal products are also a rich source of both SFAs and MUFAs. Similarly, increasing trans-fat intake across quartiles of dietary SFA might be due to the major dietary sources of trans-fats being high in dietary SFA [44]. Participants consuming on average 8\%TE SFA (Q1) also had the highest carbohydrate intake (54.1\%TE), which exceeded the recommended intake of $45-50 \% \mathrm{TE}$. It is clear from literature that replacing SFA with carbohydrate can increase fasting TAG and lower HDL-C concentrations in some population sub-groups [45, 46]. Moreover, Hooper et al. [4] reported no effect of replacing SFA with carbohydrate on CVD events and mortality in a systematic review and meta-analysis of 6 randomized controlled trials, while Schwab et al. [2] reported an increased risk of CVD outcomes in a systematic review of prospective cohort studies. Therefore, our contradictory results may be due to the higher carbohydrate intakes in the quartile which met the SFA dietary recommendation for CVD risk reduction (Q1). Interestingly, although Q2 consumed more carbohydrate than Q4, their fiber consumption was higher which might have positively influenced blood cholesterol concentrations [47]. This could suggest that the positive association of high-fat, low SFA diets on lipid risk markers might also be dependent on other dietary macronutrients and overall dietary pattern [48].

The observation that dietary SFA intake was independently associated with the fasting LDL-C concentration may be related to the impact of dietary fatty acids on LDL particle clearance. Animal and in vitro studies have suggested that dietary SFAs increase LDL-C via a downregulation in the number and expression of the hepatic LDL receptor (LDL-R) [49-51]. Although the mechanisms are still not totally understood, animal studies have provided evidence that dietary fat quality affects the LDL-R at the molecular level potentially through its effect on mRNA expression [52]. A possible explanation is that dietary SFAs lower the esterification of cholesterol in the liver by inhibiting the cholesterol esterifying enzyme acyl-CoA: cholesterol acyltransferase (ACAT), leading to increased free cholesterol accumulation which then suppress the activity of transcription factors such as sterol regulatory elementbinding proteins and liver $\mathrm{X}$ receptor, downregulating LDL-R gene expression [52-54]. In contrast, a recent study showed an increase in hepatic expression of ACAT-2 in mice fed short-term with a high SFA diet and in HepG2 cells treated with $0.5 \mathrm{mmol} / \mathrm{l}$ and $1 \mathrm{mmol} / \mathrm{l}$ palmitic acid for $14 \mathrm{~h} \mathrm{[55].} \mathrm{Furthermore,} \mathrm{it} \mathrm{has} \mathrm{been} \mathrm{argued} \mathrm{in} \mathrm{another}$ study in hamsters that increased ACAT activity may result in the formation of larger cholesterol ester enriched very 
low-density lipoprotein (VLDL) particles which may be the reason for increased LDL-C concentrations. The authors discussed that the effect of dietary fat composition on circulating cholesterol concentration might be via increased hepatic lipoprotein secretion rather than clearance [56]. Findings from an in vitro study conducted in HepG2 cells suggested that enrichment of VLDL particles with apoE following a meal rich in dietary SFA could lead to greater competition with LDL for hepatic LDL-R uptake [57]. However, these findings are from cell or animal studies, so there is a need for further studies in humans to understand the mechanisms behind the association between dietary SFAs and LDL-C concentrations.

Higher intakes of dietary SFAs have been suggested to be associated with abdominal fat accumulation, increasing CVD risk [23]. In contrast to some studies [23, 25, 58-60], we found no relationship between body fat distribution including abdominal VAT mass and SFA intake in this study. However, our findings are consistent with Greenfield et al. [61] who reported a lack of association between adiposity and dietary fat composition in their cross-sectional study in 334 female twins. This discrepancy between studies might be due to the difference in participant characteristics, study design or methods used for dietary and body composition assessments. Surprisingly, android lean mass was highest in Q3. This finding might be associated with their low carbohydrate, high SFA diet, which has previously been reported to increase lean mass, but this has only been observed in diets with high protein intakes (20-30\%TE) [62-64]. Therefore, although abdominal VAT mass explained $7 \%$ of variability in LDL-C, it was not found to be different across dietary SFA quartiles. These findings suggest that dietary SFAs and abdominal VAT may impact on LDL-C via different mechanisms.

Body fat distribution, especially abdominal VAT accumulation, has been associated with CVD risk independent of BMI, while gynoid fat is thought to be protective against metabolic diseases $[65,66]$. In the current study, fasting blood lipids (TC, TAG and LDL-C) which are established CVD risk markers, were positively associated with body fat distribution measures, including abdominal VAT mass, which confirms previous studies [67]. Furthermore, we found the TC: HDL-C ratio to explain the largest proportion of variability in abdominal VAT mass between individuals, which highlights the importance of body fat distribution in relation to CVD risk.

One proposed link between abdominal VAT and CVD is chronic and systemic inflammation, which may occur due to impaired adipocyte differentiation [68]. People with VAT accumulation have been shown to have hypertrophic dysfunctional adipocytes which release proinflammatory factors. Due to the location of abdominal
VAT, these pro-inflammatory factors can enter the liver via the portal vein and increase glucose production leading to insulin resistance, which plays a role in the development of CVD [69]. In agreement, our study showed independent associations between CRP and HOMA-IR with abdominal VAT mass, supporting previous studies showing that increased abdominal VAT leads to development of pro-inflammatory state and insulin resistance [70]. Moreover, adipocyte hypertrophy is related to decreased adiponectin levels which has been associated with increased CVD incidence [71, 72]. In line with this, in our study, adiponectin levels were negatively correlated with abdominal VAT mass and were higher in women, who were shown to have lower abdominal VAT mass compared to men. Therefore, our findings lend support to the previously reported potential mechanisms for abdominal VAT mass and CVD risk.

Strengths of this study include the large sample size, the use of DXA scans to accurately measure body fat distribution and the use of detailed dietary and physical activity assessment. Moreover, compared to the results from the current National Diet and Nutrition Survey, the dietary intake of our cohort compared closely with this representative UK population [73]. Several limitations need consideration. First, our study cannot investigate cause and effect relationship due to its observational, cross-sectional design. Furthermore, dietary intake was self-reported using a 4-day weighed food diary, therefore measurement errors are inevitable. A further limitation is that participants can under and overestimate their food intake. We have tried to address this limitation by removing the under $(n=2)$ and over $(n=3)$ reporters from the dataset for dietary analysis. In addition, as it is not always possible to exactly match the food from volunteer's diet diary with the food composition databases available, this may have influenced the dietary analysis data. Moreover, as limited data are available for $n-3$ and n- 6 PUFA on the current UK food composition databases, these dietary data should be interpreted with caution. Furthermore, dietary SFA was assessed as a single nutrient instead of the food matrix (e.g., dairy and red meat), type (e.g., palmitic and stearic acid) or source (e.g., animal or plant sources) of SFA, which may modify its effect on disease risk markers. Lastly, our study attracted individuals with a predominately normal BMI and higher physical activity level than the average UK population, therefore, it may be difficult to translate our results to the general population.

In conclusion, the findings from this cross-sectional study indicate that both dietary SFA (\%TE) and abdominal VAT mass were important determinants of the fasting LDL-C concentration. However, the lack of dose dependent relationships between quartiles of dietary SFA intake with abdominal VAT mass and LDL-C suggests that different 
mechanisms of action may exist for their impact on LDL. Therefore, further studies are needed to determine the impact of the types and sources of dietary SFA, and their relationship to abdominal obesity and CVD risk.

\begin{abstract}
Abbreviations
ACAT: Acyl-CoA: cholesterol acyltransferase; A/G fat ratio: Android to gynoid fat ratio; BMI: Body mass index; CRP: C-reactive protein; CVD: Cardiovascular disease; DBP: Diastolic blood pressure; DXA: Dual x-ray absorptiometry; $\mathrm{EE}_{\mathrm{PA}}$ : Mean energy expenditure from physical activity; GGT: Gamma-glutamyl transferase; HDL-C: High-density lipoprotein cholesterol; HOMA-IR: Homeostatic model assessment for insulin resistance; LDL-C: Low density lipoprotein cholesterol; MUFA: Monounsaturated fatty acids; NEFA: Non-esterified fatty acids; PUFA: Polyunsaturated fatty acids; SFA: Saturated fatty acid; SBP: Systolic blood pressure; TAG: Triacylglycerol; TC: Total cholesterol; VAT: Visceral adipose tissue; VAl: Visceral adiposity index; VLDL: Very low density lipoprotein; WC: Waist circumference; WHR: Waist to hip ratio; WHtR: Waist to height ratio; 25(OH)D: 25 Hydroxy vitamin D.
\end{abstract}

\section{Supplementary Information}

The online version contains supplementary material available at https://doi. org/10.1186/s12986-022-00650-y.

Additional file 1: Table S1. Spearman's correlation coefficients $\left(r_{s}\right)$ for the relationship between circulating cardiovascular disease risk markers and dietary macronutrient intakes in the whole group. Table S2. Spearman's correlation coefficients $\left(r_{s}\right)$ for the relationship between circulating cardiovascular disease risk markers and dietary macronutrient intakes in men and women. Table S3. Spearman's correlation coefficients $\left(r_{s}\right)$ for the relationship between DXA body composition measurements with CVD risk factors and dietary macronutrients in men and women.

\section{Acknowledgements}

We thank the research participants in this study and the BSc students (Hannah Ford, Liz Grey, Louise Hunter, Tsz Lau, Annie Yee Chi Poh, Rachel Pyle, Eleanor Reed, Elinor Reed, Elizabeth Stockton), MSc students (Emily Hampton, Shunying (Sharon) Huo, Juanjie Jiang, Hannah Kenney, Fatima Keshta, Shihui Liu, Natalie Lord, Ayo Ogunye, Rayan Saleh, Henry Tellwright, Lingjie Xu) and placement students (Nikki Drummond and Rebecca Finlay) for their contribution to participant recruitment and assisting with screening and study visits, sample processing and analysis. We also thank clinical unit managers Jan Luff and Sarah Hargreaves for help with recruitment and Dr. Oonagh Markey for advice on the activity monitor data collection and analysis. EO was supported by The Republic of Turkey Ministry of National Education (Ph.D. studentship).

\section{Authors' contributions}

The author responsibilities were as follows-K.G.J. and J.A.L. designed the study; E.O., R.M., S.K. and K.G.J. conducted research; MW provided advice on dietary analysis; E.O. analysed data; E.O. wrote the manuscript under the guidance of K.G.J. and J.A.L. K.G.J. had primary responsibility for final content. All the authors read and approved the final manuscript.

\section{Funding}

Not applicable.

\section{Availability of data and materials}

The datasets used and/or analysed during the current study are available from the corresponding author on reasonable request.

\section{Declarations}

\section{Ethics approval and consent to participate}

The NHS and University of Reading Research Ethics Committees (reference numbers 14/SC/1095 and 13/55, respectively) both gave a favorable ethical opinion for conduct.
Consent for publication

Not applicable.

\section{Competing interests}

The authors declare that they have no competing interests.

Received: 15 November 2021 Accepted: 31 January 2022

Published online: 03 March 2022

\section{References}

1. Penson PE, Pirro M, Banach M. LDL-C: lower is better for longer-even at low risk. BMC Med. 2020;18(1):320.

2. Schwab U, Lauritzen L, Tholstrup T, Haldorssoni T, Riserus U, Uusitupa M, et al. Effect of the amount and type of dietary fat on cardiometabolic risk factors and risk of developing type 2 diabetes, cardiovascular diseases, and cancer: a systematic review. Food Nutr Res. 2014;58:25145.

3. Mensink RP, Katan MB. Effect of dietary fatty acids on serum lipids and lipoproteins. A meta-analysis of 27 trials. Arterioscler Thromb. 1992;12(8):911-9.

4. Hooper L, Martin N, Abdelhamid A, Davey Smith G. Reduction in saturated fat intake for cardiovascular disease. Cochrane Database Syst Rev. 2020:5:Cd011737.

5. The Scientific Advisory Committee on Nutrition (SACN) report on saturated fats and health. 2019.

6. Dehghan M, Mente A, Zhang X, Swaminathan S, Li W, Mohan V, et al. Associations of fats and carbohydrate intake with cardiovascular disease and mortality in 18 countries from five continents (PURE): a prospective cohort study. Lancet. 2017;390(10107):2050-62.

7. Harcombe Z, Baker JS, DiNicolantonio JJ, Grace F, Davies B. Evidence from randomised controlled trials does not support current dietary fat guidelines: a systematic review and meta-analysis. Open heart. 2016;3(2):e000409.

8. Chowdhury R, Warnakula S, Kunutsor S, Crowe F, Ward HA, Johnson L, et al. Association of dietary, circulating, and supplement fatty acids with coronary risk: a systematic review and meta-analysis. Ann Intern Med. 2014;160(6):398-406.

9. Hruby A, Hu FB. The epidemiology of obesity: a big picture. Pharmacoeconomics. 2015;33(7):673-89.

10. Ng M, Fleming T, Robinson M, Thomson B, Graetz N, Margono C, et al. Global, regional, and national prevalence of overweight and obesity in children and adults during 1980-2013: a systematic analysis for the Global Burden of Disease Study 2013. Lancet. 2014:384(9945):766-81.

11. Zhang Y, Liu J, Yao J, Ji G, Qian L, Wang J, et al. Obesity: pathophysiology and intervention. Nutrients. 2014;6(11):5153-83.

12. Neeland IJ, Ayers CR, Rohatgi AK, Turer AT, Berry JD, Das SR, et al. Associations of visceral and abdominal subcutaneous adipose tissue with markers of cardiac and metabolic risk in obese adults. Obesity. 2013;21(9):E439-47.

13. Liu J, Fox CS, Hickson DA, May WD, Hairston KG, Carr JJ, et al. Impact of abdominal visceral and subcutaneous adipose tissue on cardiometabolic risk factors: the Jackson Heart study. J Clin Endocrinol Metab. 2010;95(12):5419-26.

14. Matsushita Y, Nakagawa T, Yamamoto S, Takahashi Y, Yokoyama T, Noda M, et al. Associations of visceral and subcutaneous fat areas with the prevalence of metabolic risk factor clustering in 6,292 Japanese individuals: the Hitachi Health Study. Diabetes Care. 2010;33(9):2117-9.

15. Shah RV, Murthy VL, Abbasi SA, Blankstein R, Kwong RY, Goldfine AB, et al. Visceral adiposity and the risk of metabolic syndrome across body mass index: the MESA Study. JACC Cardiovasc Imaging. 2014;7(12):1221-35.

16. Maki KC, Kritsch K, Foley S, Soneru I, Davidson MH. Age-dependence of the relationship between adiposity and serum low density lipoprotein cholesterol in men. J Am Coll Nutr. 1997;16(6):578-83.

17. Luo Y, Ma X, Shen Y, Hao Y, Hu Y, Xiao Y, et al. Positive relationship between serum low-density lipoprotein cholesterol levels and visceral fat in a Chinese nondiabetic population. PloS one. 2014;9(11):e112715-e.

18. Klop B, Elte JWF, Cabezas MC. Dyslipidemia in obesity: mechanisms and potential targets. Nutrients. 2013;5(4):1218-40. 
19. Zeng Q, Dong S-Y, Sun X-N, Xie J, Cui Y. Percent body fat is a better predictor of cardiovascular risk factors than body mass index. Braz J Med Biol Res Revista brasileira de pesquisas medicas e biologicas. 2012;45(7):591-600.

20. Goh LGH, Dhaliwal SS, Welborn TA, Lee AH, Della PR. Anthropometric measurements of general and central obesity and the prediction of cardiovascular disease risk in women: a cross-sectional study. BMJ Open. 2014;4(2):e004138.

21. He X, Li Z, Tang X, Zhang L, Wang L, He Y, et al. Age- and sex-related differences in body composition in healthy subjects aged 18 to 82 years. Medicine. 2018;97(25):e11152-e.

22. Álvarez-Pérez J, Sánchez-Villegas A, Díaz-Benítez EM, RuanoRodríguez C, Corella D, Martínez-González M, et al. Influence of a Mediterranean dietary pattern on body fat distribution: results of the PREDIMED-Canarias Intervention Randomized Trial. J Am Coll Nutr. 2016;35(6):568-80.

23. Rosqvist F, Iggman D, Kullberg J, Jonathan Cedernaes J, Johansson $\mathrm{H}-\mathrm{E}$, Larsson A, et al. Overfeeding polyunsaturated and saturated fat causes distinct effects on liver and visceral fat accumulation in humans. Diabetes. 2014;63:2356-68.

24. Bjermo H, Iggman D, Kullberg J, Dahlman I, Johansson L, Persson L, et al. Effects of n-6 PUFAs compared with SFAs on liver fat, lipoproteins, and inflammation in abdominal obesity: a randomized controlled trial. Am J Clin Nutr. 2012;95(5):1003-12.

25. Summers LK, Fielding BA, Bradshaw HA, llic V, Beysen C, Clark ML, et al. Substituting dietary saturated fat with polyunsaturated fat changes abdominal fat distribution and improves insulin sensitivity. Diabetologia. 2002;45(3):369-77.

26. Flock MR, Green MH, Kris-Etherton PM. Effects of adiposity on plasma lipid response to reductions in dietary saturated fatty acids and cholesterol. Adv Nutr. 2011;2(3):261-74.

27. Amato MC, Giordano C. Visceral Adiposity Index: an indicator of adipose tissue dysfunction. Int J Endocrinol. 2014;2014:730827.

28. Vanltallie TB, Yang MU, Heymsfield SB, Funk RC, Boileau RA. Heightnormalized indices of the body's fat-free mass and fat mass: potentially useful indicators of nutritional status. Am J Clin Nutr. 1990;52(6):953-9.

29. Nelson M, Atkinson M, Meyer J. A photographic atlas of food portion sizes. London: MAFF Publications; 1997.

30. Willett W. Nutritional epidemiology. Oxford: Oxford University Press; 2012.

31. Markey O, Le Jeune J, Lovegrove JA. Energy compensation following consumption of sugar-reduced products: a randomized controlled trial. Eur J Nutr. 2016;55(6):2137-49.

32. Celis-Morales CA, Perez-Bravo F, Ibañez L, Salas C, Bailey ME, Gill JM. Objective vs. self-reported physical activity and sedentary time: effects of measurement method on relationships with risk biomarkers. PloS one. 2012;7(5):e36345.

33. Troiano RP, Berrigan D, Dodd KW, Mâsse LC, Tilert T, McDowell M. Physical activity in the United States measured by accelerometer. Med Sci Sports Exerc. 2008;40(1):181-8.

34. Freedson PS, Melanson E, Sirard J. Calibration of the Computer Science and Applications, Inc. accelerometer. Med Sci Sports Exerc. 1998;30(5):777-81.

35. Friedewald WT, Levy RI, Fredrickson DS. Estimation of the concentration of low-density lipoprotein cholesterol in plasma, without use of the preparative ultracentrifuge. Clin Chem. 1972;18(6):499-502.

36. Matthews DR, Hosker JP, Rudenski AS, Naylor BA, Treacher DF, Turner RC. Homeostasis model assessment: insulin resistance and beta-cell function from fasting plasma glucose and insulin concentrations in man. Diabetologia. 1985;28(7):412-9.

37. Siri-Tarino PW, Sun Q, Hu FB, Krauss RM. Saturated fatty acids and risk of coronary heart disease: modulation by replacement nutrients. Curr Atheroscler Rep. 2010;12(6):384-90.

38. Mente A, Dehghan M, Rangarajan S, McQueen M, Dagenais G, Wielgosz $A$, et al. Association of dietary nutrients with blood lipids and blood pressure in 18 countries: a cross-sectional analysis from the PURE study. Lancet Diabetes Endocrinol. 2017;5(10):774-87.

39. Swiger KJ, Martin SS, Blaha MJ, Toth PP, Nasir K, Michos ED, et al. Narrowing sex differences in lipoprotein cholesterol subclasses following mid-life: the very large database of lipids (VLDL-10B). J Am Heart Assoc. 2014;3(2):e000851.
40. Zhang P, Su Q, Ye X, Guan P, Chen C, Hang Y, et al. Trends in LDL-C and Non-HDL-C Levels with Age. Aging Dis. 2020;11(5):1046-57.

41. Poppitt SD. Cow's milk and dairy consumption: is there now consensus for cardiometabolic health? Front Nutr. 2020;7(277).

42. RP M. Effects of saturated fatty acids on serum lipids and lipoproteins: a systematic review and regression analysis. Geneva (Switzerland). 2016. https://apps.who.int/iris/bitstream/handle/10665/246104/9789241565 349-eng.pdf. Accessed 31 Aug 2021.

43. Briggs MA, Petersen KS, Kris-Etherton PM. Saturated fatty acids and cardiovascular disease: replacements for saturated fat to reduce cardiovascular risk. Healthcare (Basel). 2017;5(2):72.

44. Dhaka V, Gulia N, Ahlawat KS, Khatkar BS. Trans fats-sources, health risks and alternative approach — a review. J Food Sci Technol. 2011;48(5):534-41.

45. Mensink RP, Zock PL, Kester AD, Katan MB. Effects of dietary fatty acids and carbohydrates on the ratio of serum total to $\mathrm{HDL}$ cholesterol and on serum lipids and apolipoproteins: a meta-analysis of 60 controlled trials. Am J Clin Nutr. 2003;77(5):1146-55.

46. Micha R, Mozaffarian D. Saturated fat and cardiometabolic risk factors, coronary heart disease, stroke, and diabetes: a fresh look at the evidence. Lipids. 2010;45(10):893-905.

47. Surampudi P, Enkhmaa B, Anuurad E, Berglund L. Lipid lowering with soluble dietary fiber. Curr Atheroscler Rep. 2016;18(12):75.

48. Jakobsen MU, O'Reilly EJ, Heitmann BL, Pereira MA, Balter K, Fraser GE, et al. Major types of dietary fat and risk of coronary heart disease: a pooled analysis of 11 cohort studies. Am J Clin Nutr. 2009;89(5):1425-32.

49. Woollett LA, Spady DK, Dietschy JM. Saturated and unsaturated fatty acids independently regulate low density lipoprotein receptor activity and production rate. J Lipid Res. 1992;33(1):77-88.

50. Mustad VA, Ellsworth JL, Cooper AD, Kris-Etherton PM, Etherton TD. Dietary linoleic acid increases and palmitic acid decreases hepatic LDL receptor protein and mRNA abundance in young pigs. J Lipid Res. 1996;37(11):2310-23.

51. Hazarika A, Kalita H, Kalita MC, Devi R. Withdrawal from high-carbohydrate, high-saturated-fat diet changes saturated fat distribution and improves hepatic low-density-lipoprotein receptor expression to ameliorate metabolic syndrome in rats. Nutrition. 2017;38:95-101.

52. Horton JD, Cuthbert JA, Spady DK. Dietary fatty acids regulate hepatic low density lipoprotein (LDL) transport by altering $L D L$ receptor protein and mRNA levels. J Clin Investig. 1993;92(2):743-9.

53. Spady DK, Woollett LA, Dietschy JM. Regulation of plasma LDL-cholesterol levels by dietary cholesterol and fatty acids. Annu Rev Nutr. 1993;13:355-81.

54. Cheema SK, Agellon LB. Metabolism of cholesterol is altered in the liver of $\mathrm{C} 3 \mathrm{H}$ mice fed fats enriched with different $\mathrm{C}-18$ fatty acids. J Nutr. 1999;129(9):1718-24.

55. Zhu-qin Z, Hou-zao C, Rui-feng Y, Ran Z, Yu-yan J, Yang X, et al. Regulation of acyl-coenzyme A: cholesterol acyltransferase 2 expression by saturated fatty acids. Chin Med Sci J Chung-kuo i hsueh k'o hsueh tsa chih. 2010;25(4):222-7.

56. Lee J-Y, Carr TP. Dietary fatty acids regulate Acyl-CoA: cholesterol acyltransferase and cytosolic cholesteryl ester hydrolase in hamsters. J Nutr. 2004;134(12):3239-44.

57. Jackson KG, Maitin V, Leake DS, Yaqoob P, Williams CM. Saturated fatinduced changes in Sf 60-400 particle composition reduces uptake of LDL by HepG2 cells. J Lipid Res. 2006;47(2):393-403.

58. Fernandez de la Puebla RA, Fuentes F, Perez-Martinez P, Sanchez E, Paniagua JA, Lopez-Miranda J, et al. A reduction in dietary saturated fat decreases body fat content in overweight, hypercholesterolemic males. Nutr Metab Cardiovasc Dis. 2003;13(5):273-7.

59. Kahleova H, Hlozkova A, Fleeman R, Fletcher K, Holubkov R, Barnard ND. Fat quantity and quality, as part of a low-fat, vegan diet, are associated with changes in body composition, insulin resistance, and insulin secretion. A 16-week randomized controlled trial. Nutrients. 2019;11(3):615.

60. Piers LS, Walker KZ, Stoney RM, Soares MJ, O'Dea K. Substitution of saturated with monounsaturated fat in a 4-week diet affects body weight and composition of overweight and obese men. Br J Nutr. 2003;90(3):717-27.

61. Greenfield JR, Samaras K, Jenkins AB, Kelly PJ, Spector TD, Campbell LV. Moderate alcohol consumption, dietary fat composition, and abdominal obesity in women: evidence for gene-environment interaction. J Clin Endocrinol Metab. 2003;88(11):5381-6. 
62. Adam-Perrot A, Clifton P, Brouns F. Low-carbohydrate diets: nutritional and physiological aspects. Obes Rev. 2006;7(1):49-58.

63. Volek JS, Sharman MJ, Love DM, Avery NG, Gómez AL, Scheett TP, et al. Body composition and hormonal responses to a carbohydrate-restricted diet. Metabolism. 2002;51(7):864-70.

64. Kirkpatrick CF, Bolick JP, Kris-Etherton PM, Sikand G, Aspry KE, Soffer DE, et al. Review of current evidence and clinical recommendations on the effects of low-carbohydrate and very-low-carbohydrate (including ketogenic) diets for the management of body weight and other cardiometabolic risk factors: a scientific statement from the National Lipid Association Nutrition and Lifestyle Task Force. J Clin Lipidol. 2019;13(5):689711.e1.

65. Després J-P, Lemieux I, Bergeron J, Pibarot P, Mathieu P, Larose E, et al. Abdominal obesity and the metabolic syndrome: contribution to global cardiometabolic risk. Arterioscler Thromb Vasc Biol. 2008;28(6):1039-49.

66. McCarty MF. A paradox resolved: the postprandial model of insulin resistance explains why gynoid adiposity appears to be protective. Med Hypotheses. 2003;61(2):173-6.

67. Shirasawa T, Ochiai H, Yoshimoto T, Nagahama S, Kobayashi M, Ohtsu I, et al. Associations between normal weight central obesity and cardiovascular disease risk factors in Japanese middle-aged adults: a cross-sectional study. J Health Popul Nutr. 2019;38(1):46.

68. Zarzour A, Kim HW, Weintraub NL. Understanding obesity-related cardiovascular disease. Circulation. 2018;138(1):64-6.

69. Longo M, Zatterale F, Naderi J, Parrillo L, Formisano P, Raciti GA, et al. Adipose tissue dysfunction as determinant of obesity-associated metabolic complications. Int J Mol Sci. 2019;20(9):2358.

70. Paniagua JA. Nutrition, insulin resistance and dysfunctional adipose tissue determine the different components of metabolic syndrome. World J Diabetes. 2016;7(19):483-514.

71. Aprahamian TR, Sam F. Adiponectin in cardiovascular inflammation and obesity. Int J Inflam. 2011;2011:3709.

72. Barrios V, Gómez-Huelgas R, Rodríguez R, de Pablos-Velasco P. Adiponectin: an emerging cardiovascular risk factor. The REFERENCE Study. Revista espanola de cardiologia. 2008;61(11):1159-67.

73. NDNS: results from years 7 and 8 [Internet]. 2018. https://www.gov.uk/ government/statistics/ndns-results-from-years-7-and-8-combined.

\section{Publisher's Note}

Springer Nature remains neutral with regard to jurisdictional claims in published maps and institutional affiliations.

- fast, convenient online submission

- thorough peer review by experienced researchers in your field

- rapid publication on acceptance

- support for research data, including large and complex data types

- gold Open Access which fosters wider collaboration and increased citations

- maximum visibility for your research: over $100 \mathrm{M}$ website views per year

At BMC, research is always in progress.

Learn more biomedcentral.com/submissions 